(a) $((a+1) / z) a+(z-3) a \quad$ when $a \equiv z-1(\bmod z)$ and $a \geqq z^{2}-5 z+3$,

(b) $[(a+1) / z](a+z)+(z-3) a$

when $a \not \equiv z-1(\bmod z)$ and $a \geqq z^{2}-4 z+2$.

We omit the proof of this result as it is rather long.

It is not hard to find the desired $N$ for specific triples of numbers. For instance when $a_{0}, a_{1}=a_{0}+2, a_{2}=a_{0}+3$ we find the value of $N$ to be $[x / 3] \cdot x+2+x$. If the largest of $a_{0}, a_{1}, a_{2}$ is sufficiently larger than the other two and those two are relatively prime then the $N$ is easily determined also. In fact if $a_{0}<a_{1}<a_{2}$ and $\left(a_{0}, a_{1}\right)=1$ and $a_{2}>\left(a_{0}-1\right)\left(a_{1}-1\right)-a_{0}$ then $N=\left(a_{0}-1\right)\left(a_{1}-1\right)$.

\title{
BIBLIOGRAPHY
}

1. A. Brauer, On a problem of partitions, Amer. J. Math. vol. 64 (1942) pp. 299312.

2. J. B. Roberts, Interaction of cycles, Bulletin of Mathematical Biophysics vol. 10 (1948) pp. 123-129.

\section{Reed College}

\section{ON THE INFINITUDE OF PRIMITIVE $k$-NONDEFICIENTS}

\section{HERBERT A. BERNHARD}

H. N. Shapiro has defined [1] a $k$-nondeficient as an integer $n$ which satisfies

$$
\sigma(n) / n \geqq k
$$

where $\sigma(n)$ is the sum of the divisors of $n$. Integers $n$ which do not satisfy (1) are called $k$-deficient. A primitive $k$-nondeficient is defined as a $k$-nondeficient, all of whose proper divisors are $k$-deficient. In the same paper, Shapiro shows that, in order for an infinite number of primitive $k$-nondeficients to exist, it is necessary that $k$ be of the form

$$
\prod_{i=1}^{m} \frac{p_{i}^{\alpha_{i}+1}-1}{\left(p_{i}-1\right) p_{i}^{\alpha_{i}}} \prod_{i=m+1}^{n} \frac{p_{i}}{p_{i}-1}
$$

or, written another way,

$$
\prod_{i=1}^{m} \frac{\sigma\left(p_{i}^{\alpha_{i}}\right)}{p_{i}^{\alpha_{i}}} \prod_{i=m+1}^{n} \frac{p_{i}}{p_{i}-1}
$$

where $p_{1}, p_{2}, p_{3}, \cdots, p_{n}$ are distinct primes and $0 \leqq m \leqq n$. In this note we show that, for every $k$ of the form (2), an infinite number of

Received by the editors May 26, 1955. 
primitive $k$-nondeficients of $n+1$ distinct prime factors do exist.

Given a $k$ of the form (2) with $n \neq m$, consider any number

$$
N_{x}=q_{x} \prod_{i=1}^{m} p_{i}^{\alpha_{i}} \prod_{i=m+1}^{n} p_{i}^{\beta_{i}}
$$

where

(a) the $p_{i}(i=1,2,3, \cdots, n)$ and the $\alpha_{i}(i=1,2,3, \cdots, m)$ are derived from $k$,

(b) the $\beta_{i}$ are chosen sufficiently large so that

$$
\begin{aligned}
\frac{\sigma\left(N_{x} / q_{x} \cdot p_{i}\right)}{N_{x} / q_{x} \cdot p_{i}} & (i=1,2,3, \cdots, m) \\
< & \frac{\sigma\left(N_{x} / q_{x} \cdot p_{i}\right)}{N_{x} / q_{x} \cdot p_{i}}(i=m+1, m+2, m+3, \cdots, n)
\end{aligned}
$$

and

(c) $q_{x}$ is a prime distinct from the $p_{i}$ and chosen sufficiently large so that $\sigma\left(N_{x}\right) / N_{x}<k$. We shall show that $N_{x}$ will become primitive $k$-nondeficient when multiplied by some factor of the form $\prod_{i=m+1}^{n} \rho_{i}^{\gamma_{i}}$, not all $\gamma_{i}=0$. And, since the choice of numbers $q_{x}$ is infinite, the process gives rise to the infinite set of primitive $k$-nondeficients we seek.

Consider the number $N_{x}(s)$ formed from $N_{x}$ by increasing each $\beta_{i}$ by some arbitrary number $s$. If $s$ is sufficiently large, then

$$
\begin{aligned}
\frac{\sigma\left[N_{x}(s)\right]}{N_{x}(s)} & \equiv \frac{q_{x}+1}{q_{x}} \prod_{i=1}^{m} \frac{p_{i}^{\alpha_{i}+1}-1}{\left(p_{i}-1\right) p_{i}^{\alpha_{i}}} \prod_{i=m+1}^{n} \frac{p_{i}^{\beta_{i}+8+1}-1}{\left(p_{i}-1\right) p_{i}^{\beta_{i}+s}} \\
& >\prod_{i=1}^{m} \frac{p_{i}^{\alpha_{i}+1}-1}{\left(p_{i}-1\right) p_{i}^{\alpha_{i}}} \prod_{i=m+1}^{n} \frac{p_{i}}{p_{i}-1} \equiv k,
\end{aligned}
$$

and $N_{x}(s)$ is $k$-nondeficient.

It is readily shown that $N_{x}(s)$ has at least one primitive $k$-nondeficient divisor. And, by condition (b) on our choice of $N_{x}$ and the fact that $\sigma\left(p^{\delta}\right) / p^{\delta}$ is less than $p /(p-1)$ for any $p, \delta$, one such divisor is of necessity a multiple of $q_{x} \prod_{i=1}^{m} p_{i}^{\alpha_{i}}$. Thus it can be found by the process of first testing for deficiency the numbers $N_{x}(s) / p_{m+1}, N_{x}(s) / p_{m+1}^{2}$, $N_{x}(s) / p_{m+1}^{3}, \cdots, N_{x}(s) / p_{m+1}^{j}, \cdots,\left(j \leqq \beta_{m+1}+s\right)$ until a deficient one is found, say $N_{x}(s) / p_{m+1}^{\eta_{1}}$. ( $\eta_{1}$ will mean $\beta_{m+1}+s+1$ if no deficient number turns up.) The same test is then applied in turn to

with powers of $p_{m+2}$, to

$$
N_{x}(s) / p_{m+1}^{\eta_{1}-1}
$$

$$
N_{x}(s) /\left(p_{m+1}^{\eta_{1}-1} \cdot p_{m+2}^{\eta_{2}-1}\right)
$$


with powers of $p_{m+3}, \cdots$, and finally to $N_{x}(s) / \prod_{i=1}^{n-m-1} p_{m+1}^{\eta_{i}-1}$ with powers of $p_{n}$. The number we seek is $N_{x}(s) / \prod_{i=1}^{n-m} p_{m+1}^{n_{i}-1}$.

For the case of a $k$ of the form (2) with $n=m$, an infinite set of primitive $k$-nondeficients is easy to find-for example, the set of all numbers $N_{x}=q_{x} \cdot \prod_{i=1}^{n} p_{i}^{\alpha_{i}}$ where the $q_{x}$ are sufficiently large to insure primitiveness and the $p_{i}, \alpha_{i}$ stem from $k$.

\section{REFERENCE}

1. Harold N. Shapiro, Note on a theorem of Dickson, Bull. Amer. Math. Soc. vol. 55 (1949) pp. 450-452.

UNIVERSITY OF MichigAN

\section{A CLASS OF SIMPLE MOUFANG LOOPS}

LOWELL J. PAIGE

1. Introduction. A Moufang loop is a loop that satisfies the associative identities

$$
x y \cdot z x=x(y z \cdot x) ; x(y \cdot x z)=(x y \cdot x) z ;(z x \cdot y) x=z(x \cdot y x) .
$$

The only known examples of simple Moufang loops are the simple groups. In the present paper we will prove the following theorem.

TheOREM. Let $R$ be a simple alternative, not-associative, ring possessing an idempotent not its unit element. Let $L$ be the loop of all regular elements of $R$ and let $Z$ be the center of $L$. Then either $L / Z$ is a simple, not-associative, Moufang loop or $L / Z$ contains a simple, not-associative, Moufang subloop $M$ which is a normal subloop of index 2.

As we shall see in the course of our proof, the present theorem is a nonassociative analogue of the well known results on the special projective group $P S L(n, K)$ (see $[4$, p. 44]).

In $\S 5$, we shall prove that the Cayley-Dickson numbers of norm 1 over the real field $R^{*}$ (modulo their center) are simple and indicate how this is the best possible result.

Our results will yield finite, not-associative, simple Moufang loops whose possible orders are $\left(2^{7 n}-2^{3 n}\right)$ and $2^{-1}\left(p^{7 n}-p^{3 n}\right)$ if $p$ is an odd prime. Thus we obtain a simple, not-associative, Moufang loop of order 120.

Although we have tried to make this paper reasonably self contained, some of the results by Bruck (2) on Moufang loops will be used without reference. 1955.

Presented to the Society, September 2, 1955; received by the editors May 30. 